\title{
Dual FLT3/TOPK inhibitor with activity against FLT3-ITD secondary mutations potently inhibits acute myeloid leukemia cell lines
}

\author{
Neetu Dayal ${ }^{\ddagger}, 1,2$, Clement Opoku-Temeng ${ }^{\ddagger 1,3}$, Delmis E Hernandez ${ }^{1,2}$, Moloud Aflaki \\ Sooreshjani ${ }^{1,2}$, Brandon A Carter-Cooper ${ }^{4}$, Rena G Lapidus ${ }^{4}$ \& Herman O Sintim*,1,2 \\ ${ }^{1}$ Purdue Institute for Drug Discovery, Department of Chemistry, Purdue University Center for Cancer Research, Purdue University, \\ West Lafayette, IN 47907, USA \\ ${ }^{2}$ Department of Chemistry, Purdue University, West Lafayette IN 47907, USA \\ ${ }^{3}$ Graduate Program in Biochemistry, University of Maryland, College Park, MD 20742, USA \\ ${ }^{4}$ University of Maryland Greenebaum Cancer Center, Baltimore, MD 21201, USA \\ * Author for correspondence: hsintim@purdue.edu \\ $\ddagger$ Authors contributed equally
}

Aim: Approximately $30 \%$ of acute myeloid leukemia (AML) patients carry FLT3 tyrosine kinase domain (TKD) mutations or internal tandem duplication (FLT3-ITD). Currently there is a paucity of compounds that are active against drug-resistant FLT3-ITD, which contains secondary mutations in the TKD, mainly at residues D835/F691. Results: HSD1169, a novel compound, is active against FLT3-ITD (D835 or F691). HSD1169 is also active against T-LAK cell-originated protein kinase (TOPK), a collaborating kinase that is highly expressed in AML cell lines. HSD1169 was active against MV4-11 and Molm-14 (FLT3-ITD cell lines) but not NOMO-1 or HL60 (FLT3-WT cell lines). HSD1169 was also active against sorafenib-resistant Molm13res cell line (containing FLT3-ITD/D835Y). Conclusion: HSD1169 or an analog could become a therapeutic agent for AML containing drug-resistant FLT3-ITD.

First draft submitted: 24 November 2017; Accepted for publication: 18 January 2018; Published online: 13 February 2018

Keywords: acute myeloid leukemia $\bullet$ drug resistance $\bullet$ FLT3-ITD • secondary mutations $\bullet$ TOPK

The human genome encodes greater than 500 protein kinases, which constitute one of the largest gene families [1]. Protein kinases control key cellular processes, including cell cycle, transcription, migration, proliferation and apoptosis via phosphotransfer [1]. Many diseases, especially cancers, are driven by mutant or aberrantly expressed protein kinases [1,2] and such kinases have become drug targets [2,3]. Imatinib (Gleevec ${ }^{\circledR}$ ) [4], an inhibitor of BCRABL and the first protein kinase inhibitor to be approved by the US FDA in 2001 for the treatment of chronic myeloid leukemia changed chronic myeloid leukemia from a lethal disease with a low 5-year survival rate to one with over 90\% survival rate. Since the imatinib breakthrough, 29 other protein kinase inhibitor drugs [5] have been approved by the FDA [5]. In majority of the cases, the administration of tyrosine kinase inhibitors (TKIs) leads to remission but ultimately relapse occurs. During relapse, the cancer driving kinase usually attains a mutation (for aberrantly expressed kinase) or secondary mutation, which reduces the affinity of the kinase for the TKI. In addition, activation of compensatory signaling pathways may occur during relapse [6]. New generation TKIs, which are active against drug-resistant kinases are now being hotly pursued.

Our group has been interested in developing compounds that inhibit the proliferation of acute myeloid leukemia (AML) as AML patients have limited therapeutic options [7,8]. The 5-year survival rate for AML is one of the lowest ( $\sim 30 \%$ overall and less than $10 \%$ for patients over 60 years) among major cancers. In particular, about a third of AML patients harboring FLT3 internal tandem duplication (FLT3-ITD) or FLT3 containing point mutation in the tyrosine kinase domain (TKD) have poor prognosis. The FLT3 kinase has therefore emerged as an important anti-AML drug target $[9,10]$. Recently, midostaurin $\left(\right.$ Rydapt $\left.^{\circledR}\right)$, a potent inhibitor of FLT3-ITD was approved as an 


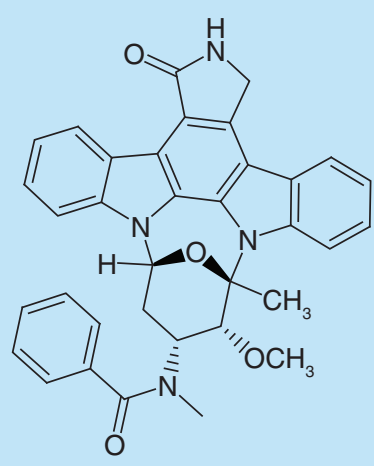

Midostaurin

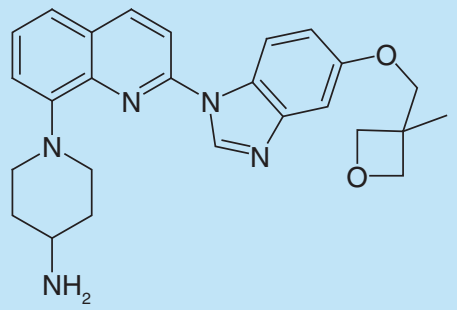

Crenolanib

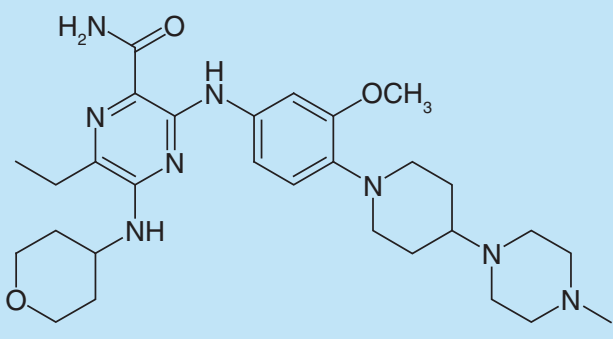

Gilteritinib

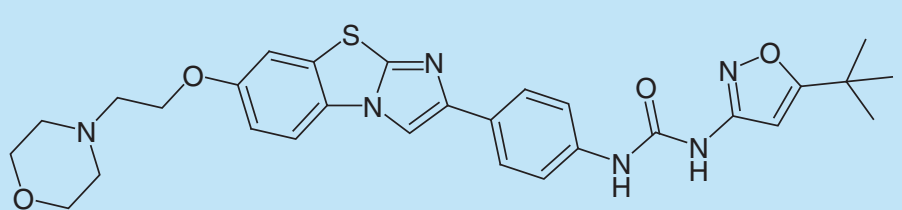

Quizartinib

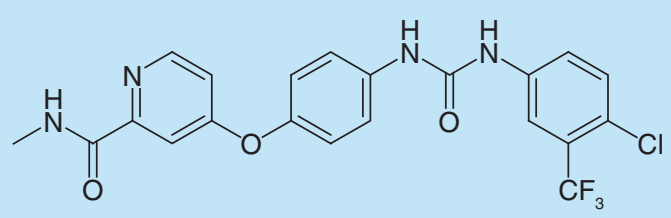

Sorafenib

Figure 1. FLT3 inhibitors in clinical trials or approved as acute myeloid leukemia drug. Midostaurin was recently approved as an AML drug. The rest are in clinical trials.

AML: Acute myeloid leukemia.

AML drug [11]. Other FLT3 inhibitors are also in different phases of clinical trials [12,13]. So far, several confounding issues regarding FLT3 inhibition as a viable strategy to cure AML remain to be resolved: midostaurin, the only approved FLT3 inhibitor so far is not a single agent and is used in combination with other cytotoxic drugs [11]; most FLT3 inhibitors, such as midostaurin, quizartinib, sorafenib and sunitinib, also inhibit c-Kit [14-17]. The concurrent inhibition of FLT3 and c-Kit leads to myelosuppression [18] but it has been challenging to develop potent FLT3 inhibitors that do not inhibit c-Kit also; most FLT3 inhibitors developed to date, such as midostaurin, ponatinib, sunitinib and sorafenib also potently inhibit VEGFRs [14,16]. VEGFRs are involved in several important processes, such as the formation and maintenance of the vasculature, platelet function, cell proliferation, migration and survival $[19,20]$. Kinase inhibitors that inhibit VEGFRs are known to cause cardiotoxicity as on-target toxicity [21] and are not ideal for elderly patients; most FLT3 inhibitors evaluated in the clinic so far do not achieve complete cure due to the emergence of secondary kinase mutations [22,23]. For example, several FLT3 inhibitors, such as quizartinib, ponatinib and sorafenib are weak inhibitors of FLT3 (D835C/H/Y) [23], which emerge only few months after FLT3 inhibitor treatment. Although there are a few FLT3 ligands that discriminate FLT3 from c-Kit (such as gilteritinib, which inhibits FLT3 with $\mathrm{IC}_{50}$ of $0.29 \mathrm{nM}$ but only inhibits c-Kit with $\mathrm{IC}_{50}$ of $230 \mathrm{nM}$ [24]) or that can inhibit certain forms of secondary mutations (e.g., crenolanib inhibits FLT3 and FLT3 D835Y with equipotent $\mathrm{IC}_{50}$ values of 1.3 and $6.9 \mathrm{nM}$, respectively [25]). So far, the discovery of an FLT3 inhibitor that solves all of the aforementioned issues has remained elusive.

In addition to FLT3 playing a central role in the pathogenesis of AML, other kinases (collaborating kinases) are also important for leukemogenesis. For example, T-LAK cell-originated protein kinase (TOPK) has been proposed as a target for AML, following the observation that it was highly expressed in most AML cell lines [26]. MV4-11 cell viability was decreased and apoptosis increased when TOPK was knocked down [26]. Furthermore, MV4-11 and MOLM13 cell lines were highly sensitive to the TOPK inhibitor OTS514 [26,27]. Based on the observations that singly targeting FLT3 or 'collaborating kinases' with inhibitors lead to pronounced growth inhibition in FLT3-driven AML cell lines, we rationalize that a multi FLT3/'collaborating kinases' inhibitor will not only have 
good potency against FLT3-ITD-driven AML but also such multitargeting inhibitor would have less propensity to encounter drug resistance, compared with an inhibitor that only targets FLT3 or the collaborating kinases. A challenge however is that the multitargeting kinase inhibitor should not inhibit 'toxicity' kinases, such as c-Kit and VEGFR, which could lead to cardiotoxicity or myelotoxicity.

\section{Experimental procedures}

\section{General procedure for synthesis of compounds}

A mixture of amine $(1 \mathrm{mmol})$ and aldehyde $(1 \mathrm{mmol})$ in $3 \mathrm{ml}$ of absolute ethanol was refluxed for $2 \mathrm{~h}$ followed by addition of cyclic ketone or acyclic ketone $(2.1 \mathrm{mmol})$ to the reaction mixture. A catalytic amount of concentrated hydrochloric acid was added and the reaction was continued to reflux for 6-12 h. After completion, sodium bicarbonate solution $(3 \mathrm{ml})$ was added to the reaction mixture and extracted with ethyl acetate $(50 \mathrm{ml} \times 2)$ and water $(25 \mathrm{ml} \times 2)$. The organic layer was collected, dried $\left(\mathrm{Na}_{2} \mathrm{SO}_{4}\right)$, concentrated under reduced pressure and purified by silica gel chromatography (dichloromethane:methanol 99:01 $\approx 80: 20$ or EtOAc:Hexane [50:50 $\approx 100: 0]$ ) to give the desired cyclized compound.

\section{Cell lines \& culture method}

MV4-11, HL60 and K562 were routinely cultured in Iscove's Modified Dulbecco's Medium, Molm13-res and Molm14 were cultured in Roswell Park Memorial Institute (RPMI) 1640 and all media were supplemented with $10 \%$ fetal bovine serum. Cells were routinely cultured at $37^{\circ} \mathrm{C}$ with $5 \% \mathrm{CO}_{2}$.

\section{Antiproliferative activity}

Molm-13-res cell line is a kind gift from Dr Sharyn Baker (The Ohio State University). MV4-11 and Molm-14 cell lines were kind gifts from Dr Mark Levis (Johns Hopkins University). HL60 and K562 were obtained from American Type Culture Collection (ATCC). Cells were seeded into 96-well microtiter plates at $2 \times 10^{4}$ cells $/ \mathrm{ml}$. Approximately $24 \mathrm{~h}$ after seeding, test compounds diluted into media were added to the cells to give a threefold serial dilution from $10 \mu \mathrm{M}$ for $\mathrm{IC}_{50}$ determination. An equal volume of DMSO diluted in medium was used as vehicle control. After treatment, the plates were incubated at $37^{\circ} \mathrm{C}$ with $5 \% \mathrm{CO}_{2}$ for $72 \mathrm{~h}$ and assayed for viability by adding CellTiter-Blue ${ }^{\circledR}$ Cell Viability Assay (Promega Corporation, WI, USA) for an additional 4 h. The plates were read with a BioTek Cytation 5 Cell Imaging Multi-Mode Reader (BioTek Instruments, VT, USA). Data were analyzed and graphed using a sigmoidal dose-response equation on GraphPad Prism 5 Software (Graphpad, CA, USA).

For NOMO-1 and MOLT-4, IC 50 was determined using the services of Reaction Biology Corporation (PA, USA). Briefly, compounds were diluted in DMSO solution with 10-dose and threefold dilutions in a source plate starting at $20 \mathrm{mM}$. Using the Echo 550 (Labcyte Inc., CA, USA), $12.5 \mathrm{nl}$ compounds were delivered into 384-well plates after which $25 \mu \mathrm{l}$ of culture media containing 2000 of NOMO-1 or MOLT- 4 cells was added to each of the wells in duplicates of the cell culture plate. The plates were incubated with the compounds at $37^{\circ} \mathrm{C}_{5} 5 \mathrm{CO}_{2}$ for $72 \mathrm{~h}$ followed by the addition of $25 \mu \mathrm{l}$ of Cell Titer Glo 2.0 reagent to each well. The contents were mixed on an orbital shaker for $2 \mathrm{~min}$ and incubated at room temperature for $15 \mathrm{~min}$ to stabilize luminescent signal. Luminescence was recorded by Envision 2104 Multilabel Reader (PerkinElmer, CA, USA). The number of viable cells in culture was determined based on quantitation of the ATP present in each culture well. The $\mathrm{IC}_{50}$ curves were plotted and $\mathrm{IC}_{50}$ values were calculated using the GraphPad Prism 4 program based on a sigmoidal dose-response equation

\section{In vitro kinase assays}

The inhibition of FLT3-ITD kinase activity by HSD1169 and related compounds were performed using the ADP-Glo ${ }^{T M}$ kinase assay system (Promega Corporation, WI, USA). Briefly, a $5 \mu$ reaction containing compounds $(100 \mathrm{nM}$ ), substrate (ATP and myelin basic protein (MBP) substrate at $10 \mu \mathrm{M}$ and $0.1 \mathrm{mg} / \mathrm{ml}$, respectively) and kinase $(30 \mathrm{nM})$ was set up in duplicates in a 384-well white plate and incubated at room temperature for $3 \mathrm{~h}$. As recommended by the manufacturer, $5 \mu$ of the ADP-Glo reagent was added for 40 min followed by the addition of $10 \mu \mathrm{l}$ of the kinase detection reagent for another $1 \mathrm{~h}$ at room temperature. Luminescence was measured using a BioTek Cytation 5 Cell Imaging Multi-Mode Reader.

The strength of binding of HSD1169 to FLT3 kinase mutants, ABL phosphorylated and nonphosphorylated was performed using the commercial KdELECT assay (DiscoverX Corporation, CA, USA) service. 


\section{Western blot analysis}

MV4-11 cells were treated with HSD1169 at the indicated concentrations or with DMSO (0.1\%). After the indicated time periods, cells were pelleted by centrifugation and lysed with M-PER ${ }^{T M}$ Mammalian Protein Extraction Reagent (Life Technologies Corporation, CA, USA) supplemented with protease inhibitor cocktail (Roche) for total protein extraction. Cells were lysed for $10 \mathrm{~min}$ on ice with gentle intermittent shaking. The cell lysates were centrifuged at $6500 \times g$ for $10 \mathrm{~min}$ at $4^{\circ} \mathrm{C}$ and the soluble proteins in the supernatant were saved. Protein concentrations of samples were determined using the bicinchoninic acid (BCA) assay. Total protein was separated on SDS-PAGE gel and transferred to a nitrocellulose membrane. The membrane was then blocked with 5\% Bovine seum albumin (BSA) in $1 \times$ TBST (Tris-buffered saline, 0.1\% Tween $20(20 \mathrm{mM}$ Tris pH7.5, $150 \mathrm{mM} \mathrm{NaCl}$ and $0.1 \%$ Tween 20)) for $1 \mathrm{~h}$ at room temperature after which primary antibodies were incubated with the membrane following the manufacturer's recommendations. The following primary antibodies from Cell Signaling (MA, USA) were used: phospho-STAT5, STAT5, TOPK and $\beta$-actin.

\section{RNA isolation \& real-time PCR analysis}

Aurum total RNA mini kit (Bio-Rad, CA, USA) was applied to extract RNA from MV4-11 cells treated with HSD 169 at the indicated concentrations for $24 \mathrm{~h}$. SuperScript ${ }^{\mathrm{TM}}$ II Reverse Transcriptase and random primers were used for the reverse transcription of the extracted RNA to cDNA. Real-time PCR was performed by QuantiTect SYBR $^{\circledR}$ Green PCR Kit and specific primers for TOPK and GADPH on a Bio-Rad CFX96 ${ }^{\circledR}$ Real-Time System (Bio-Rad, CA, USA). The data were normalized to GAPDH Ct and analyzed using the $2(-\Delta \Delta \mathrm{CT})$ method. Each condition was repeated in duplicate.

\section{Results \& discussion}

Novel chemical scaffold (8,9,10,11-tetrahydro-3H-pyrazolo[4,3-a]phenanthridine) preferentially inhibits FLT3-driven cell lines

To discover compounds that are active against both FLT3-ITD and FLT3-ITD-harboring secondary mutations in the TKD, we screened our in-house synthesized compound library for new agents that inhibit the proliferation of FLT3- and FLT3 (ITD, D835Y)-driven AML cell lines (MV4-11 (FLT3-ITD), Molm-14 (FLT3-ITD), Molm13-res (FLT3 (ITD, D835Y)) [28]. HSD1169 (see Figure 2B; synthesized via the Doebner reaction, see Figure 2A), which contains a novel 8,9,10,11-tetrahydro-3H-pyrazolo[4,3-a]phenanthridine scaffold was identified as a potent inhibitor of the three AML cell lines ( $\mathrm{IC}_{50} 5 \mathrm{nM}$ ). HSD1 169 inhibited other non-FLT3-driven leukemia cell lines (K562, NOMO-1, HL60 and MOLT4) at significantly higher concentrations (620-1855 nM, Figure 3), strongly suggesting that HSD1169 is a FLT3 inhibitor. For the discovery of cell permeable inhibitors, phenotypic screening is superior to target-based screening, as an in vitro target inhibition screening could identify potent inhibitors that may not be cell permeable or not stable in complex cell environment. Additionally, phenotypic screening could unveil compounds that have novel modes of action that could not be predicted a priori [29]. However, a limitation of phenotypic screening is that it can be time consuming to identify the target of an active compound. This limitation is not severe for compounds that inhibit cancer cell proliferation because of the availability of a panel of cancer cell lines that are driven by various cancer drivers. For example, a compound that preferentially inhibits a FLT3-driven AML but not non-FLT3-driven AML will likely act via FLT3 signaling (at least partially).

Compound HSD 169 contains five rings (labeled as A, B, C, D and E in Figure 2). Structure-activity relationship studies indicated that the ring C in HSD1169 was important for both FLT3 kinase inhibition and proliferation inhibition (Table 1). Compound 1, which lacks ring $\mathrm{C}$ is a poor inhibitor of FLT3 and did not inhibit the growth of MV4-11 (see Table 1). Compounds 2, 3, 4 (HSD1169) and 5 contain 4-, 5-, 6- and 7-membered ring C and the order of activities against FLT3 enzymatic activity and AML cell lines proliferation is HSD1169 5 $>3>>2$. This indicates that six- or seven-membered ring C is optimum for FLT3 enzymatic and AML growth inhibitions. Substituted ring $\mathrm{C}$ compounds (6 and 7) were less active (see Table 1; entries vi and vii) in inhibiting AML cell growth than HSD1169. However, the methyl-substituted ring C (compound 6) was more active than the amino-substituted ring $\mathrm{C}$ (compound 7).

We next explored how the substitution pattern of the $1 H$-pyrazole group (ring A in HSD1 169) affected both FLT3 enzymatic inhibition and AML growth inhibition. Compounds with hydrogen, methyl or trifluoromethyl groups at the 3-position of the pyrazole (compounds 8, 9 and HSD1169) were potent inhibitors of AML proliferation with the trifluoromethyl group being the best (compare entries viii, ix and iv in Table 1). Substituting the 3position of the pyrazole ring with aromatic groups (pyridyl [compound 15] or thiophen-3-yl [compound 17]) led 
(A)

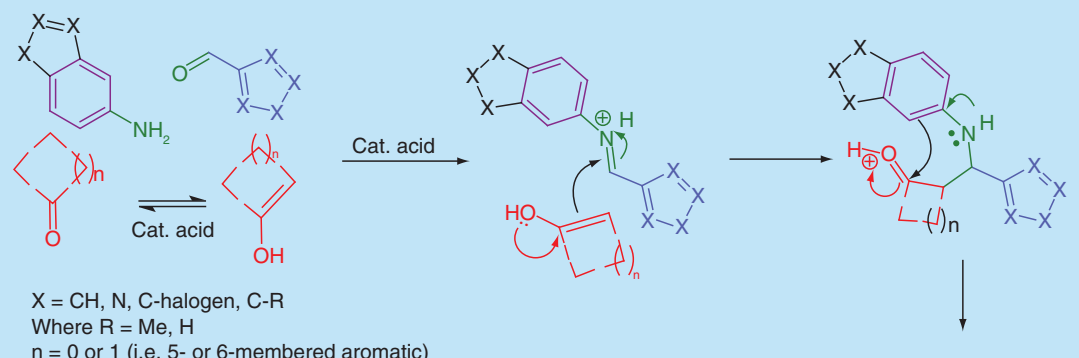

$\mathrm{n}=0$ or 1 (i.e. 5 - or 6 -membered aromatic)

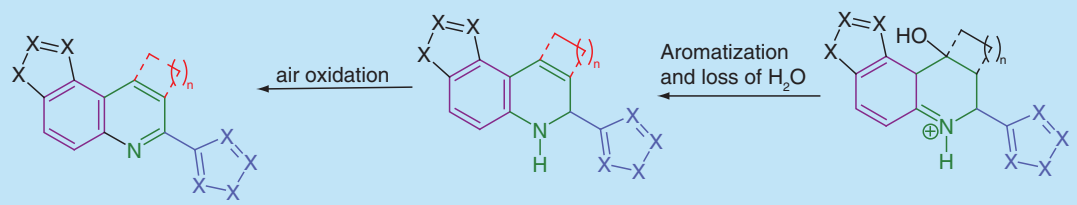

(B)

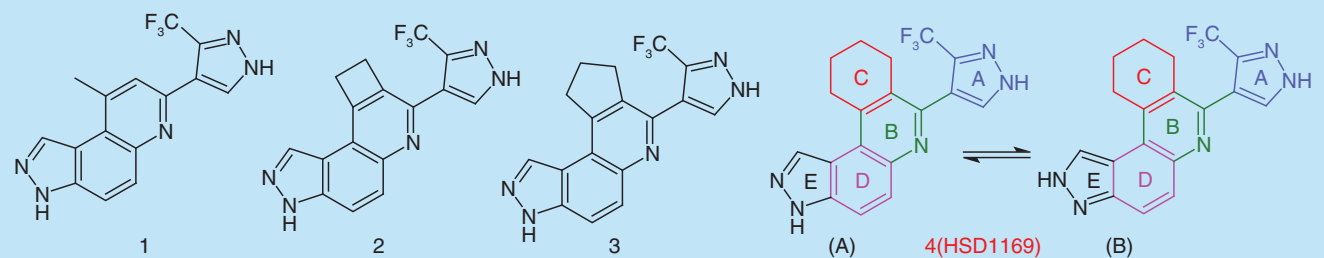<smiles>Cc1n[nH]cc1-c1nccc2[nH]ncc12</smiles>

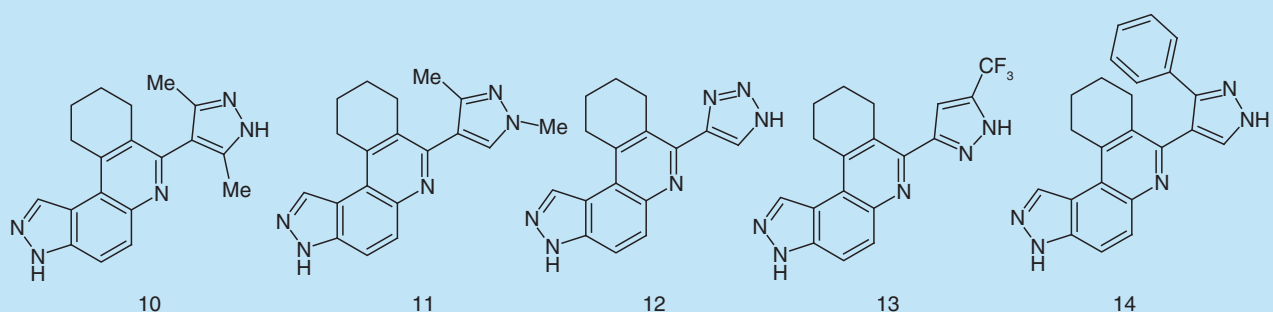<smiles>Cc1n[nH]cc1-c1nc2ccc3[nH]ccc3c2c2c1CCCC2</smiles><smiles></smiles> 


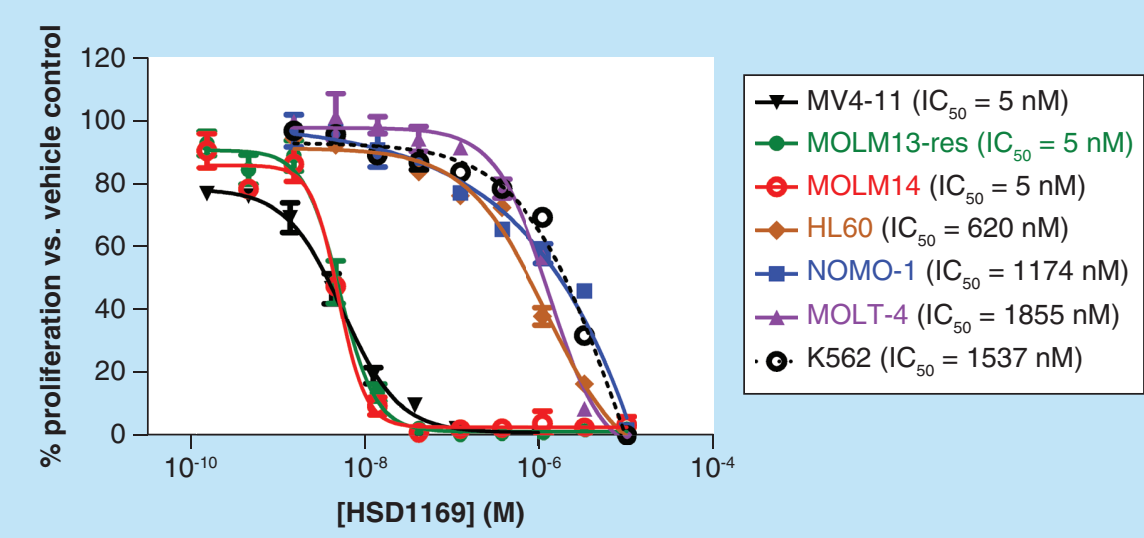

Figure 3. Dose-dependent inhibition of leukemia cell lines' proliferation by HSD1169. Plot of the dose-dependent inhibition of indicated leukemia cell lines by HSD1169. Cultures were treated with a threefold dilution starting at $10 \mu \mathrm{M}$ and incubated for $72 \mathrm{~h}$. The percent proliferation was determined relative to the DMSO control for each compound and the data were fitted to a sigmoidal dose-response equation to obtain $\mathrm{IC}_{50}$ values. Error bars represent standard error of the mean of three replicates.

\begin{tabular}{|c|c|c|c|c|c|}
\hline \multirow[t]{2}{*}{ Entry } & \multirow[t]{2}{*}{ Compound } & \multirow{2}{*}{$\begin{array}{l}\text { \%FLT3 inhibition } \\
\text { FLT3-ITD }^{\dagger}\end{array}$} & \multicolumn{3}{|c|}{$\mathrm{IC}_{50}(\mathrm{nM})$} \\
\hline & & & MV4-11 & Molm14 & Molm13-res \\
\hline 1 & 1 & 22 & 797 & ND & ND \\
\hline li & 2 & 21 & 1532 & ND & ND \\
\hline lii & 3 & 55 & 63 & 45.8 & 66.1 \\
\hline IV & 4 (HSD1169) & 80 & 5.4 & 4.9 & 5.1 \\
\hline Vi & 6 & 77 & 13.7 & 26.8 & 20.7 \\
\hline Vii & 7 & 71 & 132.5 & ND & ND \\
\hline Viii & 8 & 53 & 38.4 & 61.5 & 40.2 \\
\hline Ix & 9 & 54 & 61.5 & 64.1 & 86.7 \\
\hline$x$ & 10 & 54 & 159.4 & ND & ND \\
\hline$X v$ & 15 & 6 & 1297 & ND & ND \\
\hline Xvi & 16 & 9 & 1082 & ND & ND \\
\hline Xvii & 17 & 19 & 772 & ND & ND \\
\hline xviii & 18 & 20 & 484 & ND & ND \\
\hline Xix & 19 & 8 & 898 & ND & ND \\
\hline$X x$ & 20 & 27 & 1392 & ND & ND \\
\hline Xxi & 21 & 6 & $>5000$ & ND & ND \\
\hline
\end{tabular}

to inactive compounds. Other groups such as tert-butyl (compound 18) or carboxylic acid (compound 21) were also not tolerated at the 3-position of the pyrazole. The 1-position of the pyrazole did not tolerate substitution as compounds 11, 15, 16 and 20 with methyl, methyl, phenyl and 2-hydroxyethyl substitutions, respectively at that position were all inactive compounds (see entries xi, xv, xvi, and xx; Table 1). The 5-position of the pyrazole seems to tolerate substitution as compounds 9 (methyl group at position 3 and hydrogen at position 5) and 10 


\begin{tabular}{|c|c|c|c|c|}
\hline Kinase & HSD1169 ( Kd/nM) ${ }^{\dagger}$ & Crenolanib $(\mathrm{Kd} / \mathrm{nM})^{\ddagger}$ & Sorafenib $(\mathrm{Kd} / \mathrm{nM})^{\ddagger}$ & PKC412 (midostaurin) $(\mathrm{Kd} / \mathrm{nM})^{\S}$ \\
\hline FLT3 & 17 & 0.15 & 13 & 11 \\
\hline FLT3-ITD & 0.78 & 0.26 & 95 & 11 \\
\hline FLT3(D835H) & 4.4 & 0.16 & 11 & 6.8 \\
\hline FLT3(D835Y) & 1.1 & 0.14 & 24 & 15 \\
\hline FLT3(D835V) & 1.0 & 3.3 & 140 & - \\
\hline FLT3(ITD, D835V) & 0.41 & 3.6 & 630 & - \\
\hline FLT3(ITD, F691L) & 1.2 & 22 & 860 & - \\
\hline ABL1-phosphorylated & 7200 & 430 & - & - \\
\hline ABL1-nonphosphorylated & 5800 & 140 & 0.33 & - \\
\hline \multicolumn{5}{|c|}{$\begin{array}{l}\text { †This work: data obtained from KdELECT screening (DiscoverX). } \\
\text { †Zimmerman et al. [28]. The authors also used KdELECT screening service at DiscoverX. } \\
\text { § Reference Kd provided by DiscoverX. }\end{array}$} \\
\hline
\end{tabular}

(methyl groups at both position 3 and 5 of the pyrazole) are both active compounds (compare entries ix and x; Table 1). Compound 13 (a 3,5-substituted pyrazole) and HSD1169 (a 3,4-substituted pyrazole) only differ from each other by the substitution pattern yet HSD1169 potently inhibits AML cell lines with $\mathrm{IC}_{50}$ of $5 \mathrm{nM}$ whereas 13 is inactive even at $1000 \mathrm{nM}$. We next explored the replacement of the ring A pyrazole with other 5-membered heterocyles. Compound 12 (ring A is a triazole) was not as active as HSD1169. The nature of ring E was also critical for the inhibition of FLT3 and AML cell growth. Although HSD1169, an indazole derivative, was active, the analogous indole derivative (compound 19) did not inhibit FLT3 enzymatic activity or AML cell growth. Therefore, it appears that the different moieties of HSD1169 play important roles in the inhibition of FLT3 enzymatic activity and AML cell growth.

\section{HSD1169 potently inhibits FLT3 TKD mutants \& FLT3-ITD secondary TKD mutants}

Kinase inhibition profiling using 97 disease-relevant kinases at DiscoverX indicated that HSD1169 is a selective kinase inhibitor and only potently inhibited a handful (JAK, MKNK2 and FLT3, including FLT3 kinase mutants), see Figure 4 \& Table 2. AML patients that harbor FLT3-ITD or TKD mutation initially respond to current FLT3 inhibitors in clinical use or trial (midostaurin, sorafenib, quizartinib, crenolanib and gilteritinib) but ultimately relapse occurs due to FLT3 secondary mutation, which makes the FLT3 receptor less sensitive to the TKIs. The D835 and F691 mutations are two common mutations that occur at relapse. Quizartinib and sorafenib are not effective against both D835 and F691 secondary mutations whereas crenolanib and gilteritinib are effective against the D835Y secondary mutation. However all of the above FLT3 inhibitors are not effective against FLT3 (ITD, F691L) mutants (see Table 2). Thus there is a need for an FLT3 TKI that is effective against the F691L secondary mutants. Pleasingly, HSD1169 potently inhibits two common FLT3 secondary mutations FLT3 (ITD, D835V) and FLT3 (ITD, F691L) with Kd of 0.4 and $1.2 \mathrm{nM}$, respectively (see Table 2). We used Reaction Biology Corporation screening service to confirm that in addition to FLT3 binding, HSD1169 also potently inhibited FLT3 enzymatic activity. Consistent with the tight binding to FLT3 and mutants, HSD1169 inhibited the enzymatic activity of FLT3, FLT3-ITD and FLT3 D835Y (Figure 5). To the best of our knowledge, HSD1169 represents one of the best (if not the best) inhibitors of FLT3-ITD secondary mutation.

To have insights into the type of binding mode that HSD1169 engages with kinases, we determined the Kd for phosphorylated and nonphosphorylated ABL1. HSD1169 binds to nonphosphorylated ABL1 better (slightly) than phosphorylated ABL1 (Table 2), similar to crenolanib (a type I inhibitor) [28]. HSD1169 appears to be a type I kinase inhibitor.

FLT3 signaling, which is amplified by collaborating kinases (such as TOPK) ultimately leads to the phosphorylation of downstream effectors, such as STAT5. We therefore investigated if the administration of HSD1169 to FLT3-driven cell line, such as MV4-11 would lead to the inhibition of STAT5. In agreement with the in vitro data that show potent inhibition of FLT3, western blot analysis showed that indeed HSD1169 inhibited the phosphorylation of STAT5, see Figure 6A.

TOPK is a particularly interesting target in AML and others had shown that inhibiting TOPK causes TOPK protein downregulation, probably via the downregulation of its mRNA [26]. For example, when MV4-11 cells were treated with siRNA of TOPK, a significant decrease in mRNA and protein expression was observed [26]. 


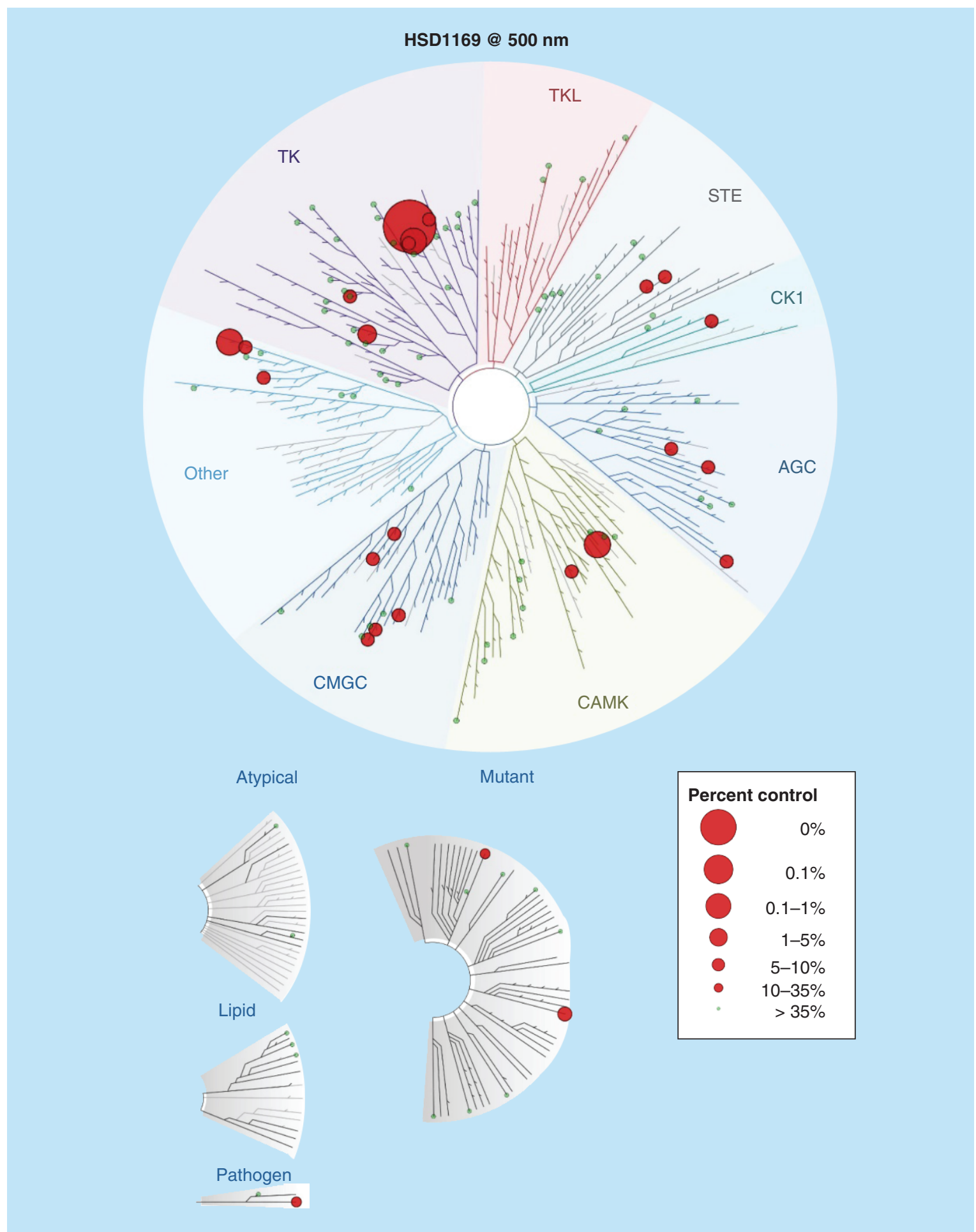

Figure 4. HSD1169 is a selective kinase inhibitor. HSD1169 has a strong affinity for FLT3, JAK2 and 3 and MKNK2 (with a percent inhibition greater than $90 \%$ at $500 \mathrm{nM}$ of HSD1169) but not other over 90 tested kinases (DiscoverX ScanEdge kinases, which are distributed across the kinome). 
Figure 5. HSD1169 potently inhibits FLT3 kinase and mutants as well as TOPK. The effect of HSD1169 on the activity of 15 nM FLT3, 100 nM FLT3 (ITD), 0.5 nM FLT3 (D835Y) and $20 \mathrm{nM}$ TOPK in the presence of $100 \mu \mathrm{M}$ ATP (done at Reaction Biology Corporation). Enzyme activity in the presence of HSD1169 was normalized to the DMSO control and data fitted to a nonlinear regression using GraphPad Prism (GraphPad, CA, USA). Error bar represent standard error of the mean of duplicate reactions.

ITD: Internal tandem duplication; TOPK: T-LAK cell-originated protein kinase.
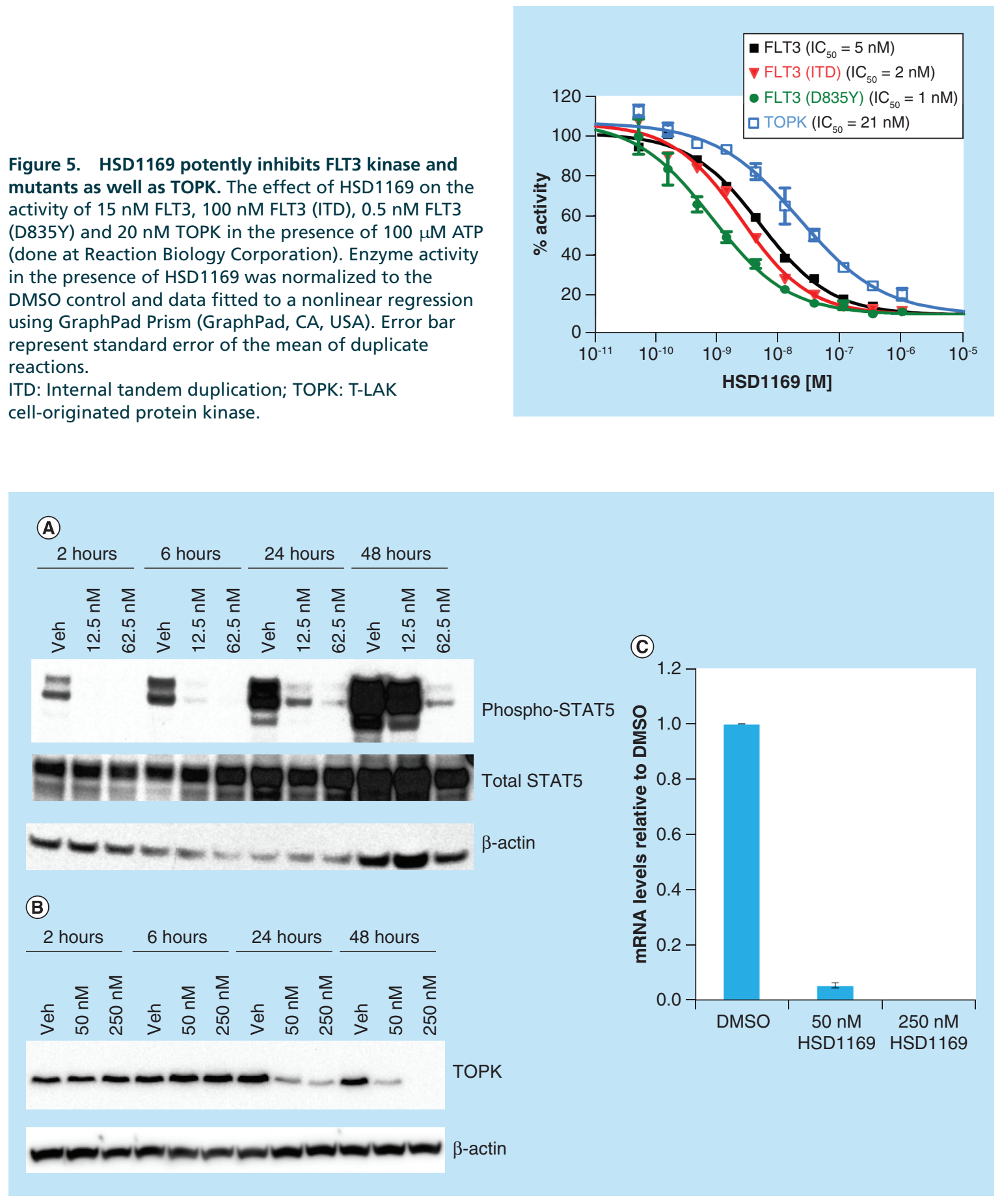

Figure 6. HSD1169 affects TOPK mRNA and protein levels in MV4-11 cells. Western blot analysis of (A) phospho-STAT5 and total STAT5, and (B) TOPK after MV4-11 cells were treated with HSD1169 at the indicated times and concentrations. (C) MV4-11 cells were treated with HSD1169 and TOPK mRNA levels were assessed after $24 \mathrm{~h}$. TOPK: T-LAK cell-originated protein kinase

We therefore investigated if HSD1169 also caused the downregulation of TOPK protein and mRNA. Consistent with the work of Alachkar et al. [26] administration of HSD1169, which is also a TOPK inhibitor (Figure 5), to MV4-11 led to the downregulation of TOPK mRNA and reduction of TOPK protein in a dose-dependent manner (Figure 6B \& C). 


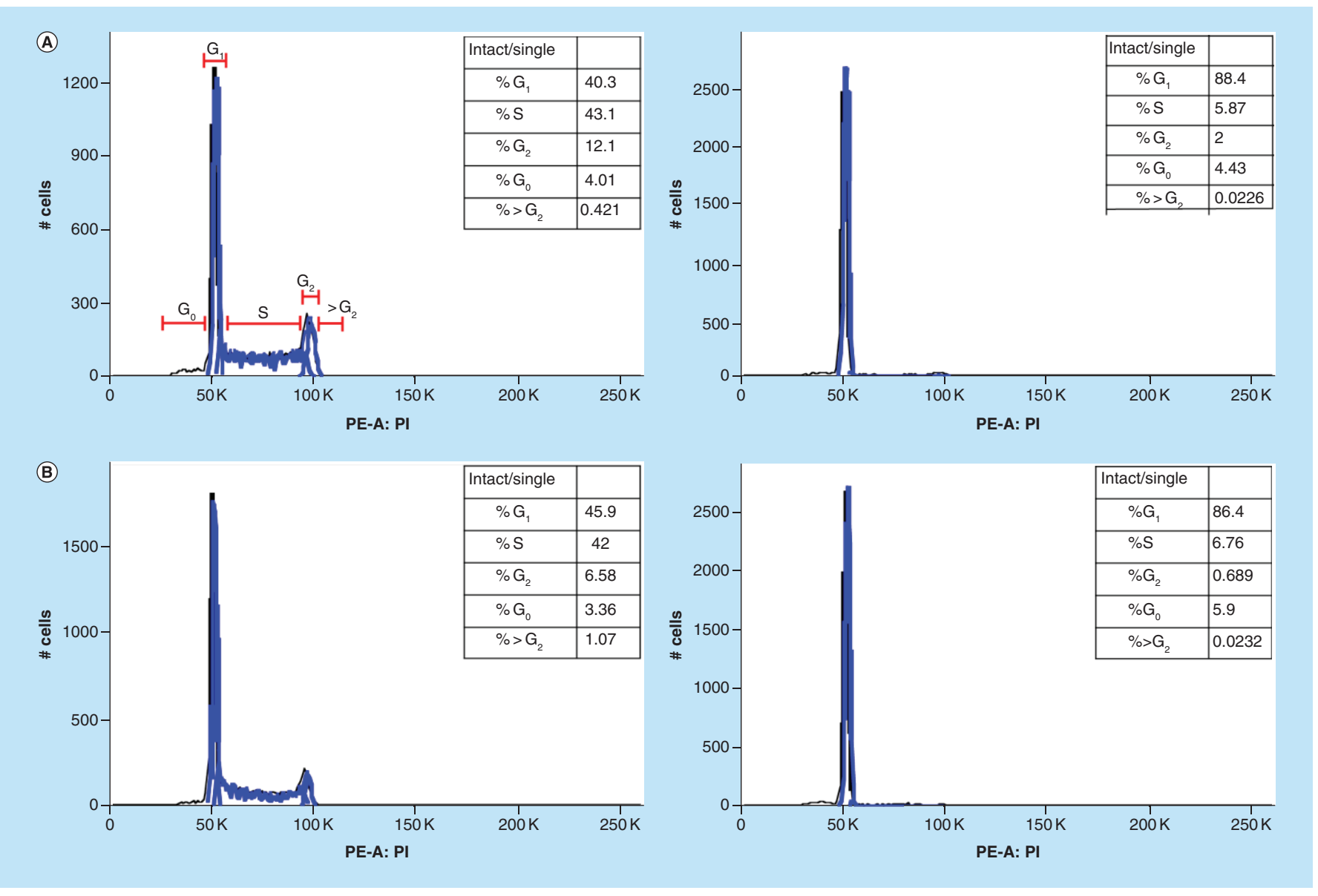

Figure 7. HSD1169 induces cell cycle arrest at the $\mathrm{G}_{0 / 1}$ phase in MV4-11 cells. Cell cycle analysis of MV4-11 before (left panels) and after (right panels) treatment with HSD1169 (62.5 nM) for (A) $24 \mathrm{~h},(B) 48 \mathrm{~h}$ and (C) $72 \mathrm{~h}$. The various phases of the cell cycle are as indicated in (A).

HSD1169 induces G1 cell cycle arrest in MV4-11 acute myeloid leukemia cells

To determine the effect of HSD1169 on cell cycle, we added different concentrations of HSD1169 to MV4-11 and the population of cells at the $G_{0}, G_{1}, S$ and $G_{2}$ phases at 24,48 and $72 \mathrm{~h}$. We observed a dose-dependent decrease in the population of cells in the $\mathrm{S}$ and G2 phases after $24 \mathrm{~h}$ and a corresponding dose-dependent increase in the percentage of cells in the $\mathrm{G}_{0 / 1}$ phase (Figure 7). Various FLT3 inhibitors cause cell cycle arrest at different phases. For example, AMG 925 (a dual FLT3/CDK4 inhibitor) [30] and AKN-028 (FLT3/CLK1/RPS6K inhibitor) [31] induce arrest in the $\mathrm{G}_{0 / 1}$ phase in Molm13 and MV 4-11 (both FLT3-ITD), respectively. Whereas PKC412 (midostaurin) did not affect cell cycle pattern in both MV4-11 and Molm13 [32]. It must be noted that in addition to FLT3, all of the reported FLT3 inhibitors also inhibit other kinases, so their effect on cell cycle will also depend on the other kinases that they inhibit.

\section{Conclusion}

In this report, we have revealed a new FLT3 inhibitor, HSD1169, which inhibits FLT3-ITD as well as the problematic D835 and F691 TKD mutants and secondary mutants FLT3 (ITD, D835V)/FLT3 (ITD, F691L). HSD1169 inhibits FLT3(ITD, F691L) with a Kd of approximately $1 \mathrm{nM}$ whereas FLT3 inhibitors in clinical trials, such as crenolanib and sorafenib, inhibit this particular mutant FLT3 with Kd of 22 and $860 \mathrm{nM}$, respectively. In addition to FLT3 inhibition, HSD1169 also inhibits collaborating kinase, TOPK, which exacerbate AML. Yet HSD1169 does not potently bind to or inhibit over 90 other kinases that were tested. The in vitro inhibition of FLT3 correlated with the antiproliferative activity of HSD1169 against FLT3-driven AML cell lines at subnanomolar 


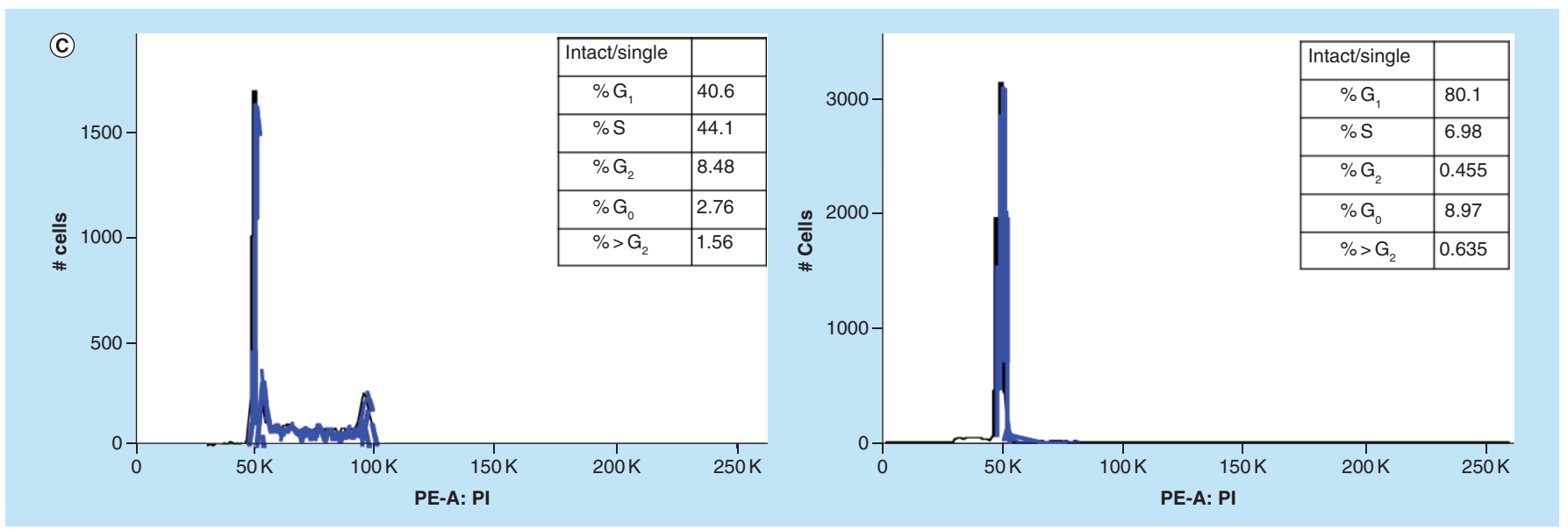

Figure 7. HSD1169 induces cell cycle arrest at the $\mathrm{G}_{0 / 1}$ phase in MV4-11 cells (cont.). Cell cycle analysis of MV4-11 before (left panels) and after (right panels) treatment with HSD1169 (62.5 nM) for (A) $24 \mathrm{~h},(B) 48 \mathrm{~h}$ and (C) $72 \mathrm{~h}$. The various phases of the cell cycle are as indicated in (A).

concentrations. We also showed that HSD1169 affected TOPK mRNA levels leading to an observed decrease in TOPK protein expression.

\section{Future perspective}

In the last few years, it has become clear that multitargeting kinase inhibitors perform better in the clinic than monotargeting inhibitors. However, a limitation of multitargeting is toxicity, if important kinases (such as VEGFR) that are relevant for essential organs such as heart and kidney are also inhibited. A challenge therefore is to develop a multitargeting but not 'dirty' kinase inhibitor (such as staurosporine, which is an overtly promiscuous kinase inhibitor and hence of limited clinical utility). We seem to have chanced upon such ideal 'multitargeting' yet clean kinase inhibitor, HSD1169. This inhibitor, HSD1169 or a close analog, represents a lead compound to develop AML patients for relapse. Ongoing work will focus on IND-enabling experiments of HSD1169 with an eye toward clinical translation.

\section{Summary points}

- FLT3-ITD kinases with secondary mutations, such as FLT3-ITD and D835/F691, are resistant to most developed FLT3 inhibitors.

- Compounds that can inhibit targets other than FLT3-ITD could potentially improve acute myeloid leukemia treatment.

- HSD1169, a very potent FLT3 inhibitor, is active against FLT3-ITD carrying TKD secondary mutations as well as TOPK.

- HSD1169 potently inhibits the proliferation of drug-resistant acute myeloid leukemia cell lines like Molm13-res.

\section{Supplementary data}

To view the supplementary data that accompany this paper please visit the journal website at:

www.future-science.com/doi/full/10.4155/fmc-2017-0298

\section{Author's contributions}

Conceptualization was done by HO Sintim. Investigation was done by N Dayal, C Opoku-Temeng, DE Hernandez, MA Sooreshjani and BA Carter-Cooper. C Opoku-Temeng helped in writing the original draft. Writing, review and editing was done by C OpokuTemeng and HO Simtim. HO Sintim helped with funding acquisition. Supervision was done by HO Simtim and RG Lapidus. 


\section{Financial \& competing interests disclosure}

The authors thank Purdue University, Purdue Cancer Center, and Elks Foundation for funding. NMR and MS data were acquired by the NMR and MS facilities supported by NIH P30 CA023168. HO Sintim and RG Lapidus are cofounders of KinaRx, a startup interested in translating AML therapeutics. The authors have no other relevant affiliations or financial involvement with any organization or entity with a financial interest in or financial conflict with the subject matter or materials discussed in the manuscript apart from those disclosed.

No writing assistance was utilized in the production of this manuscript.

\section{Ethical conduct of research}

The authors state that they have obtained appropriate institutional review board approval or have followed the principles outlined in the Declaration of Helsinki for all human or animal experimental investigations. In addition, for investigations involving human subjects, informed consent has been obtained from the participants involved.

\section{Open access}

This work is licensed under the Attribution-NonCommercial-NoDerivatives 4.0 Unported License. To view a copy of this license, visit http://creativecommons.org/licenses/by-nc-nd/4.0/

\section{References}

Papers of special note have been highlighted as: • of interest; $\bullet \bullet$ of considerable interest

1. Manning G, Whyte DB, Martinez R, Hunter T, Sudarsanam S. The protein kinase complement of the human genome. Science 298(5600), 1912-1934 (2002).

2. Fleuren EDG, Zhang L, Wu J, Daly RJ. The kinome 'at large' in cancer. Nat. Rev. Cancer 16(2), 83-98 (2016).

3. Giamas G, Stebbing J, Vorgias CE, Knippschild U. Protein kinases as targets for cancer treatment. Pharmacogenomics 8(8), 1005-1016 (2007).

4. Druker BJ. Imatinib as a paradigm of targeted therapies. Adv. Cancer Res. 91, 1-30 (2004).

5. Wu P, Nielsen TE, Clausen MH. FDA-approved small-molecule kinase inhibitors. Trends Pharmacol. Sci. 36(7), 422-439 (2015).

6. Friedman R. Drug resistance in cancer: molecular evolution and compensatory proliferation. Oncotarget 7(11), 11746-11755 (2016).

7. Ma X, Zhou J, Wang C et al. Identification of new FLT3 inhibitors that potently inhibit AML cell lines via an Azo Click-It/Staple-It approach. ACS Med. Chem. Lett. 8(5), 492-497 (2017).

8. Larocque E, Naganna N, Ma X et al. Aminoisoquinoline benzamides, FLT3 and Src-family kinase inhibitors, potently inhibit proliferation of acute myeloid leukemia cell lines. Future Med. Chem. 9(11), 1213-1225 (2017).

9. Grunwald MR, Levis MJ. FLT3 tyrosine kinase inhibition as a paradigm for targeted drug development in acute myeloid leukemia. Semin. Hematol. 52(3), 193-199 (2015).

10. Beffinger M, Skwarska A. The role of FLT3 kinase as an AML therapy target. Curr. Pharm. Des. 18(19), 2758-2765 (2012).

11. Levis M. Midostaurin approved for FLT3-mutated AML. Blood 129, 3403-3406 (2017).

12. Kiyoi H. FLT3 inhibitors: recent advances and problems for clinical application. Nagoya J. Med. Sci. 77(1-2), 7-17 (2015).

13. Wander SA, Levis MJ, Fathi AT. The evolving role of FLT3 inhibitors in acute myeloid leukemia: quizartinib and beyond. Ther. Adv Hematol. 5(3), 65-77 (2014).

14. Mendel DB, Laird AD, Xin X et al. In vivo antitumor activity of SU11248, a novel tyrosine kinase inhibitor targeting vascular endothelial growth factor and platelet-derived growth factor receptors: determination of a pharmacokinetic/pharmacodynamic relationship. Clin. Cancer Res. 9(1), 327-337 (2003).

15. Cortes JE, Kantarjian H, Foran JM et al. Phase I study of quizartinib administered daily to patients with relapsed or refractory acute myeloid leukemia irrespective of FMS-like tyrosine kinase 3-internal tandem duplication status. J. Clin. Oncol. 31(29), 3681-3687 (2013).

16. Wilhelm SM, Carter C, Tang L et al. BAY 43-9006 exhibits broad spectrum oral antitumor activity and targets the RAF/MEK/ERK pathway and receptor tyrosine kinases involved in tumor progression and angiogenesis. Cancer Res. 64(19), 7099-7109 (2004).

17. Gotlib J, Kluin-Nelemans HC, George TI et al. Efficacy and safety of midostaurin in advanced systemic mastocytosis. N. Engl. J. Med. 374(26), 2530-2541 (2016).

18. Galanis A, Levis M. Inhibition of c-Kit by tyrosine kinase inhibitors. Haematologica 100(3), e77-e79 (2015).

19. Holmes K, Roberts OL, Thomas AM, Cross MJ. Vascular endothelial growth factor receptor-2: structure, function, intracellular signalling and therapeutic inhibition. Cell Signal. 19(10), 2003-2012 (2007).

20. Cébe-Suarez S, Zehnder-Fjällman A, Ballmer-Hofer K. The role of VEGF receptors in angiogenesis; complex partnerships. Cell. Mol. Life Sci. 63(5), 601-615 (2006). 
21. Chen ZI, Ai DI. Cardiotoxicity associated with targeted cancer therapies. Mol. Clin. Oncol. 4(5), 675-681 (2016).

22. Fathi A, Levis M. FLT3 inhibitors: a story of the old and the new. Curr. Opin. Hematol. 18(2), 71-76 (2011).

23. Smith CC, Lin K, Stecula A, Sali A, Shah NP. FLT3 D835 mutations confer differential resistance to type II FLT3 inhibitors. Leukemia 29(12), 2390-2392 (2015).

24. Mori M, Kaneko N, Ueno Y et al. Gilteritinib, a FLT3/AXL inhibitor, shows antileukemic activity in mouse models of FLT3 mutated acute myeloid leukemia. Invest. New Drugs 35(5), 556-565 (2017).

25. Galanis A, Ma H, Rajkhowa $\mathrm{T}$ et al. Crenolanib is a potent inhibitor of FLT3 with activity against resistance-conferring point mutants. Blood 123(1), 94-100 (2014).

26. Alachkar H, Mutonga M, Malnassy G et al. T-LAK cell-originated protein kinase presents a novel therapeutic target in FLT3-ITD mutated acute myeloid leukemia. Oncotarget 6(32), 33410-33425 (2015).

-• Demonstrated that TOPK collaborates with FLT3-ITD in acute myeloid leukemia (AML). Also showed that TOPK was a therapeutic target in AML.

27. Matsuo Y, Park JH, Miyamoto T et al. TOPK inhibitor induces complete tumor regression in xenograft models of human cancer through inhibition of cytokinesis. Sci. Transl. Med. 6(259), 259ra145 (2014).

28. Zimmerman EI, Turner DC, Buaboonnam J et al. Crenolanib is active against models of drug-resistant FLT3-ITD-positive acute myeloid leukemia. Blood 122(22), 3607-3615 (2013).

- Crenolanib represents one of the promising AML drugs in clinical trial. It has shown activity against secondary FLT3-TKD mutations.

29. Moffat JG, Vincent F, Lee JA, Eder J, Prunotto M. Opportunities and challenges in phenotypic drug discovery: an industry perspective. Nat. Rev. Drug Discov. 16(8), 531-543 (2017).

30. Keegan K, Li C, Li Z et al. Preclinical evaluation of AMG 925, a FLT3/CDK4 dual kinase inhibitor for treating acute myeloid leukemia. Mol. Cancer Ther. 13(4), 880-889 (2014).

31. Eriksson A, Kalushkova A, Jarvius $\mathrm{M}$ et al. AKN-028 induces cell cycle arrest, downregulation of Myc associated genes and dose dependent reduction of tyrosine kinase activity in acute myeloid leukemia. Biochem. Pharmacol. 87(2), 284-291 (2014).

32. Odgerel T, Kikuchi J, Wada T et al. The FLT3 inhibitor PKC412 exerts differential cell cycle effects on leukemic cells depending on the presence of FLT3 mutations. Oncogene 27(22), 3102-3110 (2008). 
